M. Farber · D. Schütz

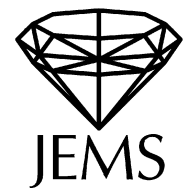

\title{
Homological category weights and estimates for $\operatorname{cat}^{1}(X, \xi)$
}

Received September 25, 2006

\begin{abstract}
In this paper we study a new notion of category weight of homology classes developing further the ideas of E. Fadell and S. Husseini [3]. In the case of closed smooth manifolds the homological category weight is equivalent to the cohomological category weight of E. Fadell and S. Husseini but these two notions are distinct already for Poincaré complexes. An important advantage of the homological category weight is its homotopy invariance. We use the notion of homological category weight to study various generalizations of the Lusternik-Schnirelmann category which appeared in the theory of closed 1 -forms and have applications in dynamics. Our primary goal is to compare two such invariants $\operatorname{cat}(X, \xi)$ and $\operatorname{cat}^{1}(X, \xi)$ which are defined similarly with reversion of the order of quantifiers. We compute these invariants explicitly for products of surfaces and show that they may differ by an arbitrarily large quantity. The proof of one of our main results, Theorem 8 uses an algebraic characterization of homology classes $z \in H_{i}(\tilde{X} ; \mathbb{Z}$ ) (where $\tilde{X} \rightarrow X$ is a free abelian covering) which are movable to infinity of $\tilde{X}$ with respect to a prescribed cohomology class $\xi \in H^{1}(X ; \mathbb{R})$. This result is established in Part II which can be read independently of the rest of the paper.
\end{abstract}

Keywords. Lusternik-Schnirelmann theory, category weight, topology of closed 1-form, homology classes movable to infinity

\section{Introduction}

In this paper we study various generalizations of the classical Lusternik-Schnirelmann category $\operatorname{cat}(X)$ which arise in topology of closed 1-forms. They are homotopy invariants of pairs $(X, \xi)$ where $X$ is a finite polyhedron and $\xi \in H^{1}(X ; \mathbb{R})$ is a real cohomology class. Several potentially different notions

$$
\operatorname{cat}(X, \xi) \leq \operatorname{cat}^{1}(X, \xi) \leq \operatorname{Cat}(X, \xi)
$$

play different roles in application of the theory of closed one-forms to dynamics (see [4], [7], [6]); each of these invariants turns into the classical cat $(X)$ when $\xi=0$. One

M. Farber and D. Schütz: Department of Mathematics, University of Durham, Durham DH1 3LE, UK; e-mail: Michael.Farber@durham.ac.uk,dirk.schuetz@durham.ac.uk

Mathematics Subject Classification (2000): Primary 55N25; Secondary 55U99 
of the objectives of the present paper is to show that cat ${ }^{1}(X, \xi)$ can be distinct from $\operatorname{cat}(X, \xi)$ and moreover their difference can be arbitrarily large. At the moment we have no examples where $\operatorname{Cat}(X, \xi)$ is distinct from $\operatorname{cat}^{1}(X, \xi)$.

It is well-known that a most effective lower bound for the classical Lusternik-Schnirelmann category $\operatorname{cat}(X)$ is the cohomological cup-length, i.e. the largest number of cohomology classes of positive degree such that their cup-product is nontrivial. In our recent preprint [9] we established cohomological cup-length type lower bounds for $\operatorname{cat}(X, \xi)$ which use local systems of a special kind. In view of (1) all lower bounds for cat $(X, \xi)$ hold for $\operatorname{cat}^{1}(X, \xi)$ as well. In order to distinguish between these two invariants one needs to have lower bounds for $\operatorname{cat}^{1}(X, \xi)$ which in general are not true for $\operatorname{cat}(X, \xi)$. Such lower bounds are found in the present paper.

Our main results are based on the idea of category weight which was initially introduced by E. Fadell and S. Husseini who proposed in [3] to attach "weights" to cohomology classes so that classes of higher weight contribute more into the cup-length estimate; see $\$ 2$ for more detail. We would like to mention also papers of Y. Rudyak [13] and J. Strom [15] who suggested a useful modification of this notion. In this paper we propose yet another variation of this idea: we attach weights to homology classes (and not to cohomology classes as did the previous authors) and measure the "level of nonvanishing" of a cup-product $u_{1} \cup \cdots \cup u_{r}$ by evaluating it $\left\langle u_{1} \cup \cdots \cup u_{r}, z\right\rangle$ on homology classes of different weight. We show that the notion of category weight of homology classes has an important advantage of being homotopy invariant (unlike the weights of Fadell and Husseini). We prove that for closed manifolds the category weight of a homology class equals the category weight of Fadell and Husseini of the dual cohomology class. We also show that this statement is false for Poincare complexes. The results about category weights of homology classes occupy Part I which can be read independently of the rest of the paper.

Part II also covers a story which may be read independently of Parts I and III. Here we study free abelian covers $p: \tilde{X} \rightarrow X$ and homology classes $z \in H_{i}(\tilde{X} ; \mathbb{Z})$ which can be realized by singular cycles lying arbitrarily far in a specified direction. Such "directions" are parametrized by cohomology classes $\xi \in H^{1}(X ; \mathbb{R})$ with $p^{*}(\xi)=0$. Our result states that this property of $z$ is equivalent to the existence of an infinite chain $c^{\prime}$ such that $\partial c^{\prime}=c$ and $c^{\prime}$ is "automatically produced out of finite data" (see the discussion after Theorem 5 . The main result of Part II generalizes Theorem 5.3 of [5] which treats the case of rank one cohomology classes. It also generalizes our previous result [8] covering the case of homology classes with coefficients in a field; in [8] our arguments use a different algebraic mechanism which fails to work over the integers.

In Part III we use the results of Parts I and II to obtain new cohomological lower bounds for $\operatorname{cat}^{1}(X, \xi)$. Our Theorem 8 gives in many cases stronger estimates than Theorem 5.6 of [5]; note that the latter theorem applies only in the special case of rank one cohomology classes although the results of the present paper are valid in full generality and do not impose this restriction. In Part III we also introduce a controlled version of $\operatorname{cat}^{1}(X, \xi)$ which behaves better under cartesian products. Finally, we compute $\operatorname{cat}^{1}(X, \xi)$ for products of surfaces as function of the cohomology class $\xi \in H^{1}(X ; \mathbb{R})$. We compare our results with the computations of the invariant $\operatorname{cat}(X, \xi)$ completed in [9]. We conclude that $\operatorname{cat}^{1}(X, \xi)$ may exceed cat $(X, \xi)$ by an arbitrarily large amount. 
The following diagram illustrates dependence of parts of this paper:

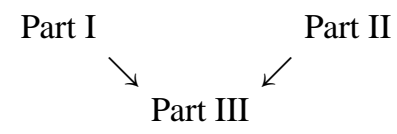

Parts I and II can be read independently, the results of Parts I \& II are used in Part III.

\section{Part I: Category weights of homology classes}

Here we introduce and study the notion of category weight of homology classes which is somewhat dual to the cohomological notion introduced by E. Fadell and S. Husseini [3]; the homological category weight has the advantage of being homotopy invariant. In Part III we use this notion to obtain improved cohomological lower bounds for $\operatorname{cat}^{1}(X, \xi)$.

\section{Basic definitions}

The classical cohomological lower bound for the Lusternik-Schnirelmann category $\operatorname{cat}(X)$ states that $\operatorname{cat}(X)>n$ if there exist $n$ cohomology classes of positive degree $u_{j} \in H^{*}\left(X ; R_{j}\right)$, where $j=1, \ldots, n$, such that their cup-product $u_{1} \ldots u_{n} \in H^{*}(X ; R)$ is nontrivial. Here $R_{j}$ denotes a local coefficient system on $X$ and $R$ is the tensor product $R_{1} \otimes \cdots \otimes R_{n}$

E. Fadell and S. Husseini [3] improved this estimate by introducing the notion of a category weight $\operatorname{cwgt}(u)$ of a cohomology class $u \in H^{q}(X ; R)$. Here is their definition:

Definition 1. Let $u \in H^{q}(X ; R)$ be a nonzero cohomology class where $R$ is a local coefficient system on $X$. One says that $\operatorname{cwgt}(u) \geq k$ (where $k \geq 0$ is an integer) if for any closed subset $A \subset X$ with cat $_{X} A \leq k$ one has $\left.u\right|_{A}=0 \in H^{q}(A ; R)$.

Recall that the inequality cat $_{X} A \leq k$ means that $A$ can be covered by $k$ open subsets $U_{i} \subset X$ such that each inclusion $U_{i} \subset X$ is null-homotopic, $i=1, \ldots, k$.

According to Definition 1 one has $\operatorname{cwgt}(u) \geq 0$ in general and $\operatorname{cwgt}(u) \geq 1$ for any nonzero cohomology class of positive degree. As Fadell and Husseini [3] showed, $\operatorname{cwgt}(u)>1$ in some special situations, which allows improving the lower estimate for cat $(X)$. Indeed, one has

$$
\operatorname{cat}(X) \geq 1+\sum_{i=1}^{n} \operatorname{cwgt}\left(u_{i}\right)
$$

assuming that the cup-product $u_{1} \ldots u_{n}$ is nonzero.

Y. Rudyak [13] and J. Strom [15] studied a modification of $\operatorname{cwgt}(u)$, called the strict category weight $\operatorname{swgt}(u)$. The latter has the advantage of being homotopy invariant. However, in some examples the strict category weight is considerably smaller than the original category weight of Fadell and Husseini. 
In this paper we introduce and exploit a "dual" notion of category weight of homology classes. It has the geometric simplicity and clarity of category weight as defined by Fadell and Husseini but has a surprising advantage of being homotopy invariant.

Definition 2. Let $z \in H_{q}(X ; R)$ be a singular homology class with coefficients in a local system $R$ and let $k \geq 0$ be a nonnegative integer. We say that $\operatorname{cwgt}(z) \geq k$ iffor any closed subset $A \subset X$ with cat $_{X} A \leq k$ there exists a singular cycle $c$ in $X-A$ representing $z$. We say that $\operatorname{cwgt}(z)=k$ iff $\operatorname{cwgt}(z) \geq k$ and $\operatorname{cwgt}(z) \geq k+1$

In other words, $\operatorname{cwgt}(z) \geq k$ is equivalent to the fact that $z$ can be realized by a singular cycle avoiding any prescribed closed subset $A \subset X$ with cat $_{X} A \leq k$.

For example, $\operatorname{cwgt}(z) \geq 1$ iff $z$ can be realized by a singular cycle avoiding any closed subset $A \subset X$ such that the inclusion $A \rightarrow X$ is homotopic to a constant map.

It will be convenient to define the category weight of the zero homology class as $+\infty$.

Formally $\operatorname{cwgt}(z) \geq k$ if $z$ lies in the intersection

$$
\bigcap_{A} \operatorname{Im}\left[H_{q}(X-A ; R) \rightarrow H_{q}(X ; R)\right]
$$

where $A \subset X$ runs over all closed subsets with cat ${ }_{X} A \leq k$.

The relation $\operatorname{cwgt}(z) \leq k$ means that there exists a closed subset $A \subset X$ with $\operatorname{cat}_{X} A \leq$ $k+1$ such that any geometric realization of $z$ intersects $A$. In particular, we obtain the following inequality:

$$
\operatorname{cat}(X) \geq \operatorname{cwgt}(z)+1
$$

for any nonzero homology class $z \in H_{q}(X ; R)$. The last inequality can also be rewritten as

$$
0 \leq \operatorname{cwgt}(z) \leq \operatorname{cat}(X)-1 \leq \operatorname{dim} X
$$

for any homology class.

Note that if $X$ is path-connected and $z$ is zero-dimensional, i.e. $z \in H_{0}(X)$, then $\operatorname{cwgt}(z)=\operatorname{cat}(X)-1$.

Lemma 1. Let $f: R \rightarrow R^{\prime}$ be a morphism of local coefficient systems over $X$ and let $f_{*}: H_{q}(X ; R) \rightarrow H_{q}\left(X ; R^{\prime}\right)$ be the induced map on homology. Then for any $z \in$ $H_{q}(X ; R)$ one has

$$
\operatorname{cwgt}\left(f_{*}(z)\right) \geq \operatorname{cwgt}(z) .
$$

Proof. The result follows directly by applying the definition.

Lemma 2. Assume that $X$ is a simplicial polyhedron. Then $\operatorname{cwgt}(z) \geq k$ iff $z$ can be realized in $X-A$ for any sub-polyhedron $A \subset X$ with cat $_{X} A \leq k$. 
Proof. We only need to show the 'if' direction. Let $A \subset X$ be closed with cat $_{X} A \leq k$. We need to show that $z$ can be realized by a cycle in $X-A$. We have $A \subset U_{1} \cup \cdots \cup U_{k}$ with each $U_{i}$ open and null-homotopic in $X$. Passing to a fine subdivision of $X$, we can find a sub-polyhedron $B \subset X$ with $A \subset B \subset U_{1} \cup \cdots \cup U_{k}$. Then cat ${ }_{X} B \leq k$ and $z$ can be realized by a cycle lying in $X-B \subset X-A$.

Example 1. Assume that $X$ is a closed 2-dimensional manifold, i.e. a compact surface. Let us show that any nonzero homology class $z \in H_{1}(X)$ has $\operatorname{cwgt}(z) \geq 1$. Indeed, it is easy to see that any closed subset $A \subset X$ which is null-homotopic in $X$ lies in the interior of a disk $D^{2} \subset X$; but $H_{1}\left(X-\operatorname{Int} D^{2}\right) \rightarrow H_{1}(X)$ is an isomorphism.

\section{Homotopy invariance of $\operatorname{cwgt}(z)$}

Lemma 3. Let $f: X \rightarrow Y$ and $g: Y \rightarrow X$ be two continuous maps with $g \circ f \simeq 1_{X}$. Let $R^{\prime}$ be a local coefficient system over $Y$ and $R=f^{*} R^{\prime}$ be the induced local system over $X$. Given a homology class $z \in H_{q}(X ; R)$, define $z^{\prime} \in H_{q}\left(Y ; R^{\prime}\right)$ by $z^{\prime}=f_{*}(z)$. Then their category weights satisfy

$$
\operatorname{cwgt}\left(z^{\prime}\right) \geq \operatorname{cwgt}(z)
$$

Proof. We start with the following well-known general remark. Let $B^{\prime} \subset Y$ be a subset which is null-homotopic in $Y$. Then the set $B=f^{-1}\left(B^{\prime}\right) \subset X$ is null-homotopic in $X$. Indeed, since $1_{X} \simeq g \circ f$, the inclusion $B \rightarrow X$ is homotopic to the composition $B \stackrel{f}{\rightarrow}$ $B^{\prime} \stackrel{i}{\rightarrow} Y \stackrel{g}{\rightarrow} X$ where the inclusion $i: B^{\prime} \rightarrow Y$ is null-homotopic by assumption.

Define $k=\operatorname{cwgt}(z)$. Assume that $A^{\prime} \subset Y$ is a closed subset with cat $A^{\prime} \leq k$. Consider $A=f^{-1}\left(A^{\prime}\right) \subset X$. Since cat $A^{\prime} \leq k$ there exist open sets $U_{1}^{\prime}, \ldots, U_{k}^{\prime} \subset Y$ covering $A^{\prime}$ with each $U_{i}^{\prime} \rightarrow Y$ null-homotopic. Then the sets $U_{i}=f^{-1}\left(U_{i}^{\prime}\right) \subset X$ are open, cover $A$ and are null-homotopic in $X$ (by the above remark). This shows that cat $_{X} A \leq k$.

Since $\operatorname{cwgt}(z) \geq k$, the class $z$ can be realized by a singular cycle in $X-A$. Then the cycle $c^{\prime}=f_{*}(c)$ in $Y$ represents the class $z^{\prime}$ and is disjoint from $A^{\prime}$ as $f$ maps $X-A$ into $Y-A^{\prime}$.

As a corollary of the previous result we obtain homotopy invariance of the category weight:

Theorem 1. If $f: X \rightarrow Y$ is a homotopy equivalence then for any homology class $z \in H_{q}(X ; R)$ one has

$$
\operatorname{cwgt}(z)=\operatorname{cwgt}\left(f_{*}(z)\right) \text {. }
$$

Here $f_{*}(z) \in H_{q}\left(X ; R^{\prime}\right)$ where $R^{\prime}=g^{*} R$ is the local coefficient system over $Y$ induced by the homotopy inverse $g: Y \rightarrow X$ of $f$. 


\section{Further properties of the category weight}

Theorem 2. Suppose that $X$ is a metric space. Assume $u \in H^{r}(X ; R), z \in H_{q}\left(X ; R^{\prime}\right)$ where $R$ and $R^{\prime}$ are local systems over $X$. Then for the homology class $u \cap z \in$ $H_{q-r}\left(X ; R \otimes R^{\prime}\right)$ one has

$$
\operatorname{cwgt}(u \cap z) \geq \operatorname{cwgt}(u)+\operatorname{cwgt}(z)
$$

Here cwgt $(z)$ is the category weight of the homology class $z$ as defined above in this paper and $\operatorname{cwgt}(u)$ is the category weight of $u$ as defined by Fadell and Husseini [3].

Proof. Write $k=\operatorname{cwgt}(z), l=\operatorname{cwgt}(u)$ and assume that $A \subset X$ is a closed subset with cat $_{X} A \leq k+l$. We want to show that $u \cap z$ can be realized in the complement $X-A$. There exists an open cover $A \subset U_{1} \cup \cdots \cup U_{k+l} \subset X$ with each $U_{i} \rightarrow X$ null-homotopic. Find open subsets $V_{i} \subset U_{i}$ such that $\bar{V}_{i} \subset U_{i}$ and $A \subset V_{1} \cup \cdots \cup V_{k+l}$.

Define $B=V_{1} \cup \cdots \cup V_{l}$ and let $C=A-B$. Clearly $C$ is closed and satisfies cat $_{X} C \leq k$. Hence $z$ can be realized by a cycle avoiding $C$. In other words, $z=i_{*}(w)$ where $w \in H_{q}\left(X-C ; R^{\prime}\right)$.

Since $\operatorname{cwgt}(u) \geq l$ we have $\left.u\right|_{B}=0$ and thus $u=j_{1}^{*}(v)$ for some $v \in H^{r}(X, B ; R)$. By statement 16 in [14, Chapter 5, §6], one has

$$
j_{*}(u \cap z)=j_{*}\left(j_{1}^{*} v \cap z\right)=v \cap \bar{j}_{*}\left(i_{*} w\right)=0
$$

where $j: X \rightarrow(X, X-A), \bar{j}: X \rightarrow(X, X-C)$ and $j_{1}: X \rightarrow(X, B)$ are inclusions. By exactness, $j_{*}(u \cap z)=0$ implies that $u \cap z$ lies in the image of $H_{q-r}\left(X-A ; R \otimes R^{\prime}\right) \rightarrow$ $H_{q-r}\left(X ; R \otimes R^{\prime}\right)$.

As a corollary we obtain:

Corollary 4. Suppose that $X$ is a metric space and for some classes $z \in H_{q}(X ; R)$ and $u \in H^{q}\left(X ; R^{\prime}\right)$ the evaluation $\langle u, z\rangle \in R^{\prime} \otimes R$ is nonzero. Then

$$
\operatorname{cat}(X) \geq \operatorname{cwgt}(z)+\operatorname{cwgt}(u)+1 .
$$

Here cwgt $(z)$ is the category weight of the homology class $z$ as defined above in this paper and $\operatorname{cwgt}(u)$ is the category weight of $u$ as defined by Fadell and Husseini [3].

Proof. This follows from inequality (2) combined with Theorem 2

Inequality (8) allows us to improve the classical cohomological lower bound for the category $\operatorname{cat}(X)$ by taking into account the quality of the homology class $z$. 


\section{Manifolds and Poincaré complexes}

In this section we prove that in the case of closed manifolds our notion of category weight coincides with the cohomological notion of Fadell and Husseini [3]. However, for Poincaré complexes these notions are distinct as we show by an example.

Theorem 3. Suppose that $X$ is a closed n-dimensional manifold, and $z \in H_{q}(X ; R)$ where $R$ is a local coefficient system. Let $u \in H^{n-q}(X ; R \otimes \tilde{\mathbb{Z}})$ be the Poincaré dual cohomology class, i.e. $z=u \cap[X]$ (see below). Then

$$
\operatorname{cwgt}(z)=\operatorname{cwgt}(u) .
$$

Here $\tilde{\mathbb{Z}}$ denotes the orientation local system on $X$, i.e. for a point $x \in X$ the stalk of $\tilde{\mathbb{Z}}$ at $x$ is $\tilde{\mathbb{Z}}_{x}=H_{n}(X, X-x ; \mathbb{Z})($ see [14]).

Proof. By the Poincaré duality theorem any homology class $z \in H_{q}(X ; R)$ can be uniquely written as $z=u \cap[X]$ where $u \in H^{n-q}(X ; R \otimes \tilde{\mathbb{Z}})$ and $[X] \in H_{n}(X ; \tilde{\mathbb{Z}})$ is the fundamental class. Applying the inequality of Theorem 2 we find

$$
\operatorname{cwgt}(z) \geq \operatorname{cwgt}(u)+\operatorname{cwgt}([X])=\operatorname{cwgt}(u) .
$$

To obtain the inverse inequality one observes that if $A \subset X$ is a closed subset with cat $_{X} A \leq \operatorname{cwgt}(z)$ then $z$ can be realized by a singular cycle in the complement $X-A$ and the usual intersection theory for chains in manifolds shows that the cocycle Poincaré dual to $z$ vanishes on $A$; hence $\operatorname{cwgt}(u) \geq \operatorname{cwgt}(z)$.

Example 2. Let $X=\mathbb{R} \mathbb{P}^{n}$ be the real projective space. For the unique nonzero homology class $z \in H_{q}\left(X ; \mathbb{Z}_{2}\right)$ one has $\operatorname{cwgt}(z)=n-q$. Indeed, the dual cohomology class is $\alpha^{n-q} \in H^{n-q}\left(X ; \mathbb{Z}_{2}\right)$ where $\alpha \in H^{1}\left(X ; \mathbb{Z}_{2}\right)$ is the generator. Clearly, $\operatorname{cwgt}\left(\alpha^{n-q}\right)=$ $n-q$.

Theorem 3 implies:

Corollary 5. If $X$ is a closed n-dimensional manifold then for any homology class $z \in$ $H_{q}(X ; R)$ with $q<n$ one has

$$
\operatorname{cwgt}(z) \geq 1 .
$$

Indeed, if $q<n$ then the dual cohomology class $u$ has positive degree and hence $\operatorname{cwgt}(u)$ $\geq 1$.

Consider now the case when $X$ is an $n$-dimensional Poincaré complex. The first part of the proof of Theorem 3 is still applicable giving inequality $(10)$ between category weights of the homology and cohomology classes. However, the second part of the proof fails. The following example shows that Theorem 3 is false for Poincare complexes. It is a modification of an argument due to D. Puppe showing that the notion of category weight of cohomology classes is not homotopy invariant. 
Example 3. Consider the lens space $L=S^{2 n+1} /(\mathbb{Z} / p)$ where $p$ is an odd prime and $\mathbb{Z} / p$ acts freely on $S^{2 n+1}$. Denote by $r: S^{2 n+1} \rightarrow L$ the quotient map. Let $X$ be the mapping cylinder of $r$, i.e.

$$
X=L \sqcup S^{2 n+1} \times[0,1] / \sim
$$

where each point $(x, 0) \in S^{2 n+1} \times[0,1]$ is identified with $r(x) \in L$. Clearly $X$ is homotopy equivalent to $L$ and so it is a Poincaré complex. By a theorem of Krasnosel'skii [10], the category of $X$ equals $2 n+2$. Hence for $z=1 \in H_{0}\left(X ; \mathbb{Z}_{2}\right)$ one has

$$
\operatorname{cwgt}(z)=\operatorname{cat}(X)-1=2 n+1
$$

(see above). The dual cohomology class $u$ is the generator $u \in H^{2 n+1}\left(X ; \mathbb{Z}_{2}\right)$. Let us show that

$$
\operatorname{cwgt}(u)=1 \text {. }
$$

Indeed, consider the sphere $S=S^{2 n+1} \times 1 \subset X$. The restriction $\left.u\right|_{S} \in H^{2 n+1}\left(S ; \mathbb{Z}_{2}\right)$ coincides with the induced class $r^{*}(v)$ where $v \in H^{2 n+1}\left(L ; \mathbb{Z}_{2}\right)$ is the generator. Hence the cohomology class $\left.u\right|_{S}$ is nonzero. However, the sphere $S$ has category 2 and moreover cat $_{X} S=2$ (as the inclusion $S \rightarrow X$ is not null-homotopic).

The following simple construction gives non-manifolds for which the category weight can be explicitly calculated.

Lemma 6. Let $X=X_{1} \vee X_{2}$ be the wedge of two polyhedra $X_{1}$ and $X_{2}$ and let $z \in$ $H_{q}(X ; R)$ be the sum $z=z_{1}+z_{2}$ where $z_{i} \in H_{q}\left(X_{i} ; R_{i}\right)$ and $R_{i}=\left.R\right|_{X_{i}}$. Then

$$
\operatorname{cwgt}(z)=\min \left\{\operatorname{cwgt}\left(z_{1}\right), \operatorname{cwgt}\left(z_{2}\right)\right\} .
$$

Here $\operatorname{cwgt}\left(z_{i}\right)$ is the category weight of $z_{i}$ viewed as a homology class of $X_{i}$.

Proof. The inequality $\operatorname{cwgt}(z) \leq \min \left\{\operatorname{cwgt}\left(z_{1}\right), \operatorname{cwgt}\left(z_{2}\right)\right\}$ is obvious. Let $A \subset X$ be a closed subset with $\operatorname{cat}_{X} A \leq k$ where $k=\min \left\{\operatorname{cwgt}\left(z_{1}\right), \operatorname{cwgt}\left(z_{2}\right)\right\}$. Then $A=A_{1} \vee A_{2}$ where $A_{i} \subset X_{i}$ and cat $X_{i} A_{i} \leq k$, where $i=1,2$. One can realize $z_{i}$ by a cycle avoiding $A_{i}$. The sum of these two cycles is a cycle representing $z$ which avoids $A$. Thus we obtain the opposite inequality $\operatorname{cwgt}(z) \geq k$.

\section{Strict category weight}

The notion of strict category weight was introduced in [13]; it is a homotopy invariant variation of the category weight of Fadell and Husseini [3]. We use this notion in this paper and therefore recall the relevant definitions. We warn the reader that our terminology differs from [13] by 1 and is consistent with [3].

Definition 3. Given a continuous map $\phi: A \rightarrow X$, we say that $\operatorname{cat}(\phi) \leq k$ if $A$ can be covered by $k$ open sets $A_{1}, \ldots, A_{k}$ such that each restriction $\left.\phi\right|_{A_{i}}$ is null-homotopic. The strict category weight of a cohomology class $u \in H^{q}(X ; R)$ (where $R$ is a local coefficient system on $X)$ is defined as the maximal integer $k$ such that $\phi^{*}(u)=0$ for any continuous map $\phi: A \rightarrow X$ with $\operatorname{cat}(\phi) \leq k$. 
The strict category weight is denoted by $\operatorname{swgt}(u)$. Clearly, one has

$$
\operatorname{swgt}(u) \leq \operatorname{cwgt}(u)
$$

and $\operatorname{swgt}(u) \geq 1$ for any cohomology class $u \in H^{q}(X ; R)$ of positive degree $q>0$.

Definition 4. Let $X$ be a closed smooth connected $n$-dimensional manifold. We define the strict category weight of a homology class $z \in H_{q}(X ; R)$ (denoted $\left.\operatorname{swgt}(z)\right)$ as the strict category weight of the dual cohomology class $u \in H^{n-q}(X ; R \otimes \tilde{\mathbb{Z}})$.

A similar definition can be used in the case of Poincaré complexes, but we do not use it in such generality.

Proposition 7. Let $z_{i} \in H_{q_{i}}\left(X_{i} ; R_{i}\right)$ where $X_{i}$ is a closed smooth orientable manifold of dimension $n_{i}, i=1,2$. Consider the cross-product

$$
z_{1} \times z_{2} \in H_{q}\left(X_{1} \times X_{2} ; R\right)
$$

where $q=q_{1}+q_{2}$ and $R$ is the external tensor product $R=R_{1} \otimes R_{2}$. Then

$$
\operatorname{swgt}\left(z_{1} \times z_{2}\right) \geq \operatorname{swgt}\left(z_{1}\right)+\operatorname{swgt}\left(z_{2}\right) .
$$

Proof. Let $u_{i} \in H^{n_{i}-q_{i}}\left(X_{i} ; R_{i}\right)$ denote the dual of $z_{i}$, where $i=1,2$. Then the dual of $z_{1} \times z_{2}$ is $u_{1} \times u_{2} \in H^{n-q}\left(X_{1} \times X_{2} ; R\right)$ where $n=n_{1}+n_{2}$. Consider also the classes $u_{1} \times 1 \in H^{n_{1}-q_{1}}\left(X_{1} \times X_{2} ; R_{1} \otimes \mathbb{Z}\right)$ and $1 \times u_{2} \in H^{n_{2}-q_{2}}\left(X_{1} \times X_{2} ; \mathbb{Z} \otimes R_{2}\right)$.

Define $k_{i}=\operatorname{swgt}\left(z_{i}\right)=\operatorname{swgt}\left(u_{i}\right)$. Let $\phi: A \rightarrow X_{1} \times X_{2}$ be a continuous map with $\operatorname{cat}(\phi) \leq k_{1}+k_{2}$. Then $A$ is the union of open subsets $A=A_{1} \cup A_{2}$ such that $\left.\operatorname{cat}(\phi)\right|_{A_{i}} \leq k_{i}$. We that $\left.\phi^{*}\left(u_{1} \times 1\right)\right|_{A_{1}}=0$ and $\left.\phi^{*}\left(1 \times u_{2}\right)\right|_{A_{2}}=0$. This implies that the class $\phi^{*}\left(u_{1} \times u_{2}\right)=\phi^{*}\left(u_{1} \times 1\right) \cup \phi^{*}\left(1 \times u_{2}\right)$ vanishes. Hence $\operatorname{swgt}\left(z_{1} \times z_{2}\right) \geq k_{1}+k_{2}$.

Corollary 8. Let $X_{i}$ be closed orientable manifolds and $z_{i} \in H_{q_{i}}\left(X_{i} ; R_{i}\right)$ where $q_{i}<$ $\operatorname{dim} X_{i}$ for $i=1, \ldots, k$. Consider $z=z_{1} \times \cdots \times z_{k} \in H_{q}(X ; R)$ where $X=X_{1} \times \cdots \times X_{k}$, $q=q_{1}+\cdots+q_{k}$ and $R=R_{1} \otimes \cdots \otimes R_{k}$. Then

$$
\operatorname{cwgt}(z) \geq k
$$

This corollary is a source of examples of homology classes having high category weight.

\section{Part II: Moving integral homology classes to infinity}

In Part II we study conditions for an integral homology class $z \in H_{i}(\tilde{X} ; \mathbb{Z})$ of a free abelian covering $\tilde{X} \rightarrow X$ to be movable to infinity with respect to a cohomology class $\xi \in H^{1}(X ; \mathbb{R})$. The case of homology classes with coefficients in a field was studied in [8] using a different algebraic technique which is not applicable over $\mathbb{Z}$. 


\section{Abel-Jacobi maps and neighbourhoods of infinity}

For the convenience of the reader we recall in this section the language introduced in [8]. Let $X$ be a connected finite cell complex and $p: \tilde{X} \rightarrow X$ a regular covering having a free abelian group of covering transformations $H \simeq \mathbb{Z}^{r}$. Write $H_{\mathbb{R}}=H \otimes \mathbb{R}$; it is a vector space of dimension $r$ containing $H$ as a lattice.

Proposition 9. There exists a canonical Abel-Jacobi map

$$
A: \tilde{X} \rightarrow H_{\mathbb{R}}
$$

having the following properties:

(a) A is $H$-equivariant; here $H$ acts on $\tilde{X}$ by covering transformations and it acts on $H_{\mathbb{R}}$ by translations.

(b) A is proper (i.e. the preimage of a compact subset of $H_{\mathbb{R}}$ is compact).

(c) $A$ is determined uniquely up to replacing it by a map $A^{\prime}: \tilde{X} \rightarrow H_{\mathbb{R}}$ of the form $A^{\prime}=A+F \circ p$ where $F: X \rightarrow H_{\mathbb{R}}$ is a continuous map.

This fact is well-known; we refer to [8] for a detailed proof.

Let $\xi \in H^{1}(X ; \mathbb{R})$ be a cohomology class with the property

$$
p^{*}(\xi)=0 \in H^{1}(\tilde{X} ; \mathbb{R}) .
$$

Such a class $\xi$ can be viewed either as a homomorphism $\xi: H \rightarrow \mathbb{R}$ or as a linear functional $\xi_{\mathbb{R}}: H_{\mathbb{R}} \rightarrow \mathbb{R}$.

Definition 5. A subset $N \subset \tilde{X}$ is called a neighbourhood of infinity in $\tilde{X}$ with respect to the cohomology class $\xi$ if $N$ contains the set

$$
\left\{x \in \tilde{X} ; \xi_{\mathbb{R}}(A(x))>c\right\} \subset N,
$$

for some real $c \in \mathbb{R}$. Here $A: \tilde{X} \rightarrow H_{\mathbb{R}}$ is an Abel-Jacobi map for the covering $p: \tilde{X} \rightarrow X$

See [8] for more details.

\section{Homology classes movable to infinity}

Let $G$ be an abelian group (the coefficient system). We mainly have in mind the cases of $G=\mathbb{Z}$ or $G=\mathbf{k}$ a field.

Definition 6 (see [4, §5]). A homology class $z \in H_{i}(\tilde{X} ; G)$ is said to be movable to infinity of $\tilde{X}$ with respect to a nonzero cohomology class $\xi \in H^{1}(X ; \mathbb{R}), p^{*}(\xi)=0$, if in any neighbourhood $N$ of infinity with respect to $\xi$ there exists a (singular) cycle with coefficients in $G$ representing $z$. 
Equivalently, a homology class $z \in H_{i}(\tilde{X} ; G)$ is movable to infinity with respect to $\xi \in$ $H^{1}(X ; \mathbb{R})$ if $z$ lies in the intersection

$$
\bigcap_{N} \operatorname{Im}\left[H_{i}(N ; G) \rightarrow H_{i}(\tilde{X} ; G)\right]
$$

where $N$ runs over all neighbourhoods of infinity in $\tilde{X}$ with respect to $\xi$. This can also be expressed by saying that $z$ lies in the kernel of the natural homomorphism

$$
H_{i}(\tilde{X} ; G) \rightarrow \lim _{\leftarrow} H_{i}(\tilde{X}, N ; G)
$$

where in the inverse limit $N$ runs over all neighbourhoods of infinity in $\tilde{X}$ with respect to $\xi$.

The following theorem proven in [8] gives an explicit description of all movable homology classes in the case when $G=\mathbf{k}$ is a field. It generalizes the result of [4, §5] treating the simplest case of infinite cyclic covers $q: \tilde{X} \rightarrow X$.

Theorem 4. Let $X$ be a finite cell complex and $q: \tilde{X} \rightarrow X$ be a regular covering having a free abelian group of covering transformations $H \simeq \mathbb{Z}^{r}$. Let $\xi \in H^{1}(X ; \mathbb{R})$ be $a$ nonzero cohomology class of rank $r$ satisfying $q^{*}(\xi)=0$. The following properties of a nonzero homology class $z \in H_{i}(\tilde{X} ; \mathbf{k})$ (where $\mathbf{k}$ is a field) are equivalent:

(A) $z$ is movable to infinity with respect to $\xi$.

(B) Any singular cycle $c$ in $\tilde{X}$ realizing the class $z$ bounds an infinite singular chain $c^{\prime}$ in $\tilde{X}$ containing only finitely many simplices lying outside every neighbourhood of infinity $N \subset \tilde{X}$ with respect to $\xi$.

(C) There exists a nonzero element $x \in \mathbf{k}[H]$ such that $x \cdot z=0$.

Later in this paper (see $\$ 9$ ) we will describe the set of homology classes with integral coefficients which are movable to infinity.

\section{Integral homology classes movable to infinity}

To get an analogue of Theorem 4 in the case of integral coefficients, we need another definition.

Definition 7. Let $H$ be a group and $\xi: H \rightarrow \mathbb{R}$ a homomorphism. A nonzero element $\Delta \in \mathbb{Z}[H]$ is said to have $\xi$-lowest coefficient 1 if $\Delta=(1-y) h$ with $h \in H$ and $y=\sum a_{j} g_{j}$, where the $g_{j} \in H$ satisfy $\xi\left(g_{j}\right)>0$ and $a_{j} \in \mathbb{Z}$.

Theorem 5. Let $X$ be a finite cell complex and $p: \tilde{X} \rightarrow X$ be a regular covering having a free abelian group of covering transformations $H \simeq \mathbb{Z}^{r}$. Let $\xi \in H^{1}(X ; \mathbb{R})$ be a nonzero cohomology class of rank $r$ satisfying $p^{*}(\xi)=0$. The following properties of a nonzero integral homology class $z \in H_{i}(\tilde{X} ; \mathbb{Z})$ are equivalent:

(A) $z$ is movable to infinity with respect to $\xi$. 
(B) Any singular cycle $c$ in $\tilde{X}$ realizing the class $z$ bounds an infinite singular chain $c^{\prime}$ in $\tilde{X}$ with integral coefficients containing only finitely many simplices lying outside every neighbourhood of infinity $N \subset \tilde{X}$ with respect to $\xi$.

(C) There exists a nonzero element $\Delta \in \mathbb{Z}[H]$ with $\xi$-lowest coefficient 1 such that $\Delta \cdot z=0$.

This result improves Theorem 5.3 of [5] which treats the case of rank one cohomology classes, $r=1$. Movability to infinity of homology classes with coefficients in a field was studied in [4] ( $r=1$ case) and in [8] $(r \geq 1)$.

Note that the implications $(\mathrm{C}) \Rightarrow(\mathrm{B}) \Rightarrow(\mathrm{A})$ of Theorem 5 are straightforward (see below); the only nontrivial statement is the implication $(\mathrm{A}) \Rightarrow(\mathrm{C})$. Let us explain why $(\mathrm{C}) \Rightarrow(\mathrm{B})$. Suppose that $\Delta \cdot z=0 \in H_{i}(\tilde{X} ; \mathbb{Z})$ where $\Delta \in \mathbb{Z}[H]$ has $\xi$-lowest coefficient 1 . Without loss of generality we may assume that $\Delta=1-y$ where $y \in \mathbb{Z}[H]$ is $\xi$-positive, i.e. $y$ is a finite sum of the form $\sum a_{j} g_{j}$ where $g_{j} \in H, \xi\left(g_{j}\right)>0$, and $a_{j} \in \mathbb{Z}$. Let $c$ be a chain representing the class $z$. Then the cycle $\Delta \cdot c$ bounds, i.e. $(1-y) \cdot c=\partial c_{1}$ where $c_{1}$ is a finite chain in $\tilde{X}$. Set $c^{\prime}=c_{1}+y c_{1}+y^{2} c_{1}+\cdots$. Then $\partial c^{\prime}=c$ and $c^{\prime}$ has finitely many simplices lying outside every neighbourhood of infinity $N \subset \tilde{X}$ with respect to $\xi$.

The main part of the proof consists in establishing the vanishing of the $\lim ^{1}$ term in the following exact sequence:

$$
\left.0 \rightarrow \lim ^{1} H_{q+1}(\tilde{X}, N ; \mathbb{Z}) \rightarrow H_{q}(X ; \widehat{\mathbb{Z}[H]}]_{\xi}\right) \rightarrow \lim _{\leftarrow} H_{q}(\tilde{X}, N ; \mathbb{Z}) \rightarrow 0 .
$$

This exact sequence was described in $\S 6$ of [8]. Formally, the proof of the exactness of [19] given in [8] assumes that the ring of coefficients is a field but it works equally well in the case $\mathbb{Z}$ with no modifications. In the exact sequence $19 \mathrm{lim}$ and $\lim ^{1}$ are taken relative to the system of neighbourhoods of infinity $N \subset \tilde{X}$ with respect to $\xi$. The symbol $\widehat{\mathbb{Z}[H]}]_{\xi}$ in ${ }_{19}$ denotes the Novikov completion of the group ring $\mathbb{Z}[H]$ (see [11], [12]). Recall that elements of the group ring $\mathbb{Z}[H]$ are finite sums of the form $\sum a_{i} g_{i}$ where $a_{i} \in \mathbb{Z}$ and $g_{i} \in H$; the ring $\left.\widehat{\mathbb{Z}[H]}\right]_{\xi}$ also includes all countable sums $\sum a_{i} g_{i}$ having the property $\lim _{i \rightarrow+\infty} \xi\left(g_{i}\right)=+\infty$.

Proposition 10. Under the conditions of Theorem 5 one has

$$
\lim ^{1} H_{q}(\tilde{X}, N ; \mathbb{Z})=0
$$

where $N$ runs over all neighbourhoods of infinity in $\tilde{X}$ with respect to $\xi$ partially ordered by reverse inclusion.

Proposition 10 gives the implication $(\mathrm{A}) \Rightarrow(\mathrm{B})$ of Theorem 5 Indeed, using Definition 6 combined with 20 we see that a homology class $z \in H_{q}(X ; \mathbb{Z})$ is movable to infinity with respect to $\xi$ if and only if a cycle $c \in C_{q}(\tilde{X})$ representing $z$ bounds a chain $c^{\prime} \in$ $\left.C_{q}(\tilde{X}) \otimes \widehat{\mathbb{Z}[H]}\right]_{\xi}$, i.e. $\partial c^{\prime}=c$. Here $C_{*}(\tilde{X})$ denote the cellular chain complex of $\tilde{X}$ with 
integral coefficients. One can view $c^{\prime}$ as an infinite chain in $\tilde{X}$ having finitely many terms outside any given neighbourhood of infinity in $\tilde{X}$ with respect to $\xi$.

To see that $(\mathrm{B}) \Rightarrow(\mathrm{C})$, let $S_{\xi} \subset \mathbb{Z}[H]$ be the subset consisting of elements with $\xi$ lowest coefficient 1 and $\Lambda_{\xi}=S_{\xi}^{-1} \mathbb{Z}[H]$ the localization. By [6, Lemma 1.13] the inclusion $\Lambda_{\xi} \rightarrow \widehat{\mathbb{Z}[H]_{\xi}}$ is faithfully flat so that the change of coefficients $H_{*}\left(X ; \Lambda_{\xi}\right) \rightarrow$ $H_{*}\left(X ; \widehat{\mathbb{Z}[H]}_{\xi}\right)$ is injective as well. The result follows.

\section{Proof of Theorem 5}

First we discuss some commutative algebra. Recall our notations. $H=\mathbb{Z}^{r}$ is a free abelian group and $\xi: H \rightarrow \mathbb{R}$ is an injective group homomorphism. We denote by $A$ the Novikov ring $\widehat{\mathbb{Z}}_{[H]}$ and by $A_{0}$ its subring $\widetilde{\mathbb{Z}\left[H_{0}\right]} \xi_{\xi}$ where $H_{0}=\{g \in H ; \xi(g) \geq 0\}$. Elements of $A_{0}$ are countable formal sums of the form $\sum_{j} a_{j} g_{j}$ where $a_{j} \in \mathbb{Z}$ and $\xi\left(g_{j}\right)$ tends to $+\infty$.

It is well-known that $A$ is a principal ideal domain but $A_{0}$ is not. Our goal is to obtain some partial results about properties of modules over the ring $A_{0}$ resembling those of modules over principal ideal domains.

Definition 8. Let $M$ be an $A_{0}$-module. A sequence of elements $m_{1}, \ldots, m_{k} \in M$ is a quasi-basis for $M$ if (1) for any $m \in M$ there exists $g \in H_{0}$ such that gm can be represented in the form $g m=\sum a_{j} m_{j}$ where $a_{j} \in A_{0}$, and (2) there are no nontrivial relations $\sum a_{j} m_{j}=0$.

Lemma 11. Let $f: A_{0}^{n} \rightarrow A_{0}^{m}$ be a homomorphism of finitely generated free $A_{0}$ modules. Then there exist quasi-bases $d_{1}, \ldots, d_{n} \in A_{0}^{n}$ and $e_{1}, \ldots, e_{m} \in A_{0}^{m}$ and an integer $\mu \leq \min \{n, m\}$ such that for any $j \leq \mu$ one has

$$
f\left(d_{j}\right)=a_{j} e_{j}, \quad \text { where } a_{j} \in A_{0}, a_{j} \neq 0,
$$

and $f\left(d_{j}\right)=0$ for $j>\mu$.

Proof. Localizations of $A_{0}^{n}$ and $A_{0}^{m}$ with respect to the multiplicative set $H$ lead to free modules over the principal ideal domain $A$. Hence, applying the standard theory, we find free bases $d_{1}^{\prime}, \ldots, d_{n}^{\prime} \in A^{n}$ and $e_{1}^{\prime}, \ldots, e_{m}^{\prime} \in A^{m}$ and an integer $\mu \leq \max \{n, m\}$ such that $f\left(d_{j}^{\prime}\right)=a_{j}^{\prime} e_{j}^{\prime}$ for $j \leq \mu$ (where $a_{j}^{\prime} \in A, a_{j}^{\prime} \neq 0$ ) and $f\left(d_{j}^{\prime}\right)=0$ for $j>\mu$. Choose $g \in H_{0}$ such that $g d_{j}^{\prime} \in A_{0}^{n}$ and $g e_{j}^{\prime} \in A_{0}^{m}$ for all $j$. Choose $g^{\prime} \in H_{0}$ such that $g^{\prime} a_{j}^{\prime} \in A_{0}$ for all $j$. Now set $d_{j}=g g^{\prime} d_{j}^{\prime}, e_{j}=g e_{j}^{\prime}$, and $a_{j}=g^{\prime} a_{j}^{\prime}$. We obtain quasi-bases $d_{j}$ and $e_{j}$ and clearly the relations 21$]$ hold.

Lemma 12. Let $C_{*}$ be a free finitely generated 1 chain complex over $A_{0}$. Then there exist quasi-bases $e_{1}^{q}, e_{2}^{q}, \ldots, e_{r_{q}}^{q} \in C_{q}$ (where $q \in \mathbb{Z}$ and $r_{q}$ denotes the rank of $C_{q}$ ) and

\footnotetext{
1 By this we mean that each $A_{0}$-module $C_{q}$ is finitely generated and only finitely many modules $C_{q}$ are nonzero.
} 
integers $\mu_{q} \leq \min \left\{r_{q}, r_{q-1}\right\}$ such that the differential $d: C_{q} \rightarrow C_{q-1}$ is given by

$$
d\left(e_{j}^{q}\right)= \begin{cases}a_{j}^{q} e_{j}^{q-1} & \text { for } j \leq \mu_{q}, \\ 0 & \text { for } j>\mu_{q},\end{cases}
$$

and the elements $a_{j}^{q} \in A_{0}$ are nonzero.

Proof. The proof essentially repeats the arguments of Lemma 11. In the first step we construct a basis $f_{j}^{q}$ of the localized chain complex $C_{q}^{\prime}=A \otimes_{A_{0}} C_{q}$ over the principal ideal domain $A$ such that all differentials $d: C_{q}^{\prime} \rightarrow C_{q-1}^{\prime}$ have the diagonal form $d\left(f_{j}^{q}\right)=\alpha_{j}^{q} f_{j}^{q-1}$ with $\alpha_{j}^{q} \in A$. In the second step one multiplies the basis $f_{j}^{q}$ by a suitable group element $g^{q} \in H_{0}$ so that (1) the elements $e_{j}^{q}=g^{q} f_{j}^{q}$ lie in the original complex $C_{q}$ and (2) the elements $a_{j}^{q}=g^{q}\left(g^{q-1}\right)^{-1} \alpha_{j}^{q}$ lie in $A_{0}$.

Lemma 13. Let $C_{*}$ be a free finitely generated chain complex over $A_{0}$. Then there exists a finitely generated free chain subcomplex $D_{*} \subset C_{*}$ such that $g C_{*} \subset D_{*}$ for some group element $g \in H_{0}$ and $H_{j}\left(D_{*}\right)$ is isomorphic to a finite direct sum of cyclid $A_{0}$-modules.

Proof. Apply Lemma 12 and take for $D_{q} \subset C_{q}$ the $A_{0}$-submodule generated by the elements $e_{1}^{q}, \ldots, e_{r_{q}}^{q}$.

Next we apply the above results to obtain the following corollary.

Corollary 14. Let $C_{*}$ be a free finitely generated chain complex over $A_{0}$. Let $\bar{C}_{*}=$ $A \otimes{ }_{A_{0}} C_{*}$ be the localized chain complex and $i: C_{*} \rightarrow \bar{C}_{*}$ the inclusion. Then for any $q$ there exists a group element $g=g^{q} \in H_{0}$ such that the kernel of the induced map $i_{*}: H_{q}(C) \rightarrow H_{q}(\bar{C})$ coincides with the kernel of multiplication by $g$ on $H_{q}(C)$.

Proof. As a preparation, consider a nonzero cyclic $A_{0}$-module $M_{0}=A_{0} /\left(a A_{0}\right)$ and the associated $A$-module $M=A /(a A)$. Here $a \in A_{0}$ is a noninvertible element. Write $a$ in the form $a=g(\alpha+h \beta)$ where $g \in H_{0}, \alpha \in \mathbb{Z}, \alpha \neq 0, \beta \in A_{0}$ and $h \in H$ is such that $\xi(h)>0$. Note that $M$ is trivial if and only if $a$ is invertible in $A$, i.e. when $\alpha= \pm 1$. Similarly, $M_{0}$ is trivial iff $a$ is invertible in $A_{0}$, i.e. when $g=0$ and $\alpha= \pm 1$. We will say that $M_{0}$ is a cyclic module of the first (second) kind if $\alpha= \pm 1$ (or $|\alpha|>1$, respectively). We see that for a cyclic module $M_{0}$ of the first kind there exists $g \in H_{0}$ such that $g M_{0}=0$ and the corresponding module $M$ is trivial. For a cyclic module $M_{0}$ of the second kind, there is a $g \in H$ such that $g M_{0} \rightarrow M$ is injective. Indeed, with $a=g(\alpha+h \beta)$ as above and $M_{0}=A_{0} /\left(a A_{0}\right)$, we get $g M_{0}=A_{0} /\left(\alpha+h \beta A_{0}\right)$, which injects into $A /(\alpha+h \beta A)$.

Apply Lemma 13 to obtain a subcomplex $D_{*} \subset C_{*}$ such that $g^{\prime} C_{*} \subset D_{*}$ for some $g^{\prime} \in H_{0}$ and $H_{*}(D)$ is a finite direct sum of cyclic $A_{0}$-modules. One finds that $A \otimes_{A_{0}} D_{*}=$ $A \otimes_{A_{0}} C_{*}=\bar{C}_{*}$ and $H_{q}(\bar{C})=A \otimes_{A_{0}} H_{q}(C)=A \otimes_{A_{0}} H_{q}(D)$ since $A$ is flat over $A_{0}$. Also, the kernel of the map restricted to the summands of cyclic modules of the second

\footnotetext{
2 That is, modules of the form $A_{0} /\left(a A_{0}\right)$ where $a \in A_{0}$
} 
kind can be annihilated by multiplication with a suitable element of $H_{0}$. Thus there exists an $h \in H_{0}$ such that the kernel of the map $H_{q}(D) \rightarrow H_{q}(\bar{C})$ coincides with the kernel of $h: H_{q}(D) \rightarrow H_{q}(D)$.

Now set $g=h g^{\prime} \in H_{0}$. Let us show that the kernel of $i_{*}: H_{q}(C) \rightarrow H_{q}(\bar{C})$ coincides with the kernel of multiplication $g_{*}: H_{q}(C) \rightarrow H_{q}(C)$ by $g$. Consider the following diagram:

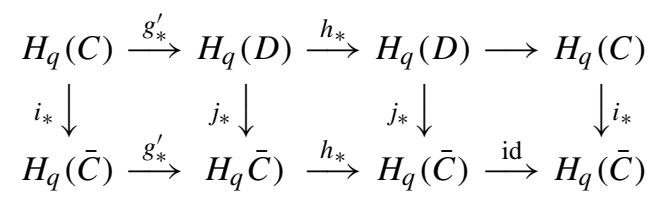

The composition of the upper horizontal row is multiplication by $g$, i.e. the map $g_{*}$ : $H_{q}(C) \rightarrow H_{q}(C)$. Every map appearing in the lower horizontal row is an isomorphism. From the previous paragraph we know that $\operatorname{Ker}\left(j_{*}\right)=\operatorname{Ker}\left(h_{*}\right)$. Therefore, examining the diagram, we find that $\operatorname{Ker}\left(i_{*}\right)=\operatorname{Ker}\left(g_{*}\right)$ as claimed.

The vanishing of the $\lim ^{1}$ term of the exact sequence 19 (i.e. Proposition 10 , see above) would follow once one has the Mittag-Lefler condition (see [16, Prop. 3.5.7]), which in our case states:

Proposition 15. For any neighbourhood of infinity $N \subset \tilde{X}$ with respect to $\xi$ there exists a neighbourhood of infinity $N^{\prime} \subset N$ such that for any neighbourhood of infinity $N^{\prime \prime} \subset N^{\prime}$ one has

$$
\operatorname{Im}\left[H_{q}\left(\tilde{X}, N^{\prime \prime}\right) \rightarrow H_{q}(\tilde{X}, N)\right]=\operatorname{Im}\left[H_{q}\left(\tilde{X}, N^{\prime}\right) \rightarrow H_{q}(\tilde{X}, N)\right] .
$$

The homology groups appearing in 23 are with coefficients in $\mathbb{Z}$ and all neighbourhoods of infinity are with respect to a fixed cohomology class $\xi$.

The equality 23 can be expressed by saying that any cycle in $\tilde{X}$ relative to $N$ which can be refined to a cycle relative to $N^{\prime}$ can be refined to a cycle relative to an arbitrary neighbourhood of infinity $N^{\prime \prime} \subset N^{\prime} \subset \tilde{X}$.

Proof of Proposition 15 Let $C_{*}(\tilde{X})$ denote the cellular chain complex of $\tilde{X}$. It is a complex of finitely generated free $\mathbb{Z}[H]$-modules. Let $N \subset \tilde{X}$ be a cellular neighbourhood of infinity with respect to $\xi$ as described in Lemma 3 of [8]. The cellular chain complex $C_{*}(N)$ is free and finitely generated over $\mathbb{Z}\left[H_{0}\right]$ where $H_{0}=\{g \in H ; \xi(g) \geq 0\}$. Consider the completed chain complexes $C_{*}^{\prime}(N)=A_{0} \otimes_{\mathbb{Z}\left[H_{0}\right]} C_{*}(N)$ and $C_{*}^{\prime}(\tilde{X})=$ $A \otimes_{\mathbb{Z}[H]} C_{*}(\tilde{X})$. Recall that $\left.A=\widehat{\mathbb{Z}[H]}\right]_{\xi}$ is the Novikov ring and $A_{0}={\widehat{\mathbb{Z}\left[H_{0}\right]_{\xi}}}_{\xi}$. The canonical inclusions $C_{*}(N) \rightarrow C_{*}^{\prime}(N)$ and $C_{*}(\tilde{X}) \rightarrow C_{*}^{\prime}(\tilde{X})$ determine a chain homomorphism

$$
F: C_{*}(\tilde{X}) / C_{*}(N) \stackrel{\simeq}{\rightarrow} C_{*}^{\prime}(\tilde{X}) / C_{*}^{\prime}(N)
$$

which is an isomorphism. Injectivity of $F$ is equivalent to $C_{*}(\tilde{X}) \cap C_{*}^{\prime}(N)=C_{*}(N)$ (which is obvious) and surjectivity of $F$ is equivalent to $C_{*}(\tilde{X})+C_{*}^{\prime}(N)=C_{*}^{\prime}(\tilde{X})$. The latter follows from the equality $\mathbb{Z}[H]+A_{0}=A$ for subrings of $A$. 
The short exact sequence of chain complexes over $A_{0}$,

$$
0 \rightarrow C_{*}^{\prime}(N) \rightarrow C_{*}^{\prime}(\tilde{X}) \rightarrow C_{*}(\tilde{X}) / C_{*}(N) \rightarrow 0,
$$

gives the exact sequence

$$
\cdots \rightarrow H_{q}^{\prime}(N) \stackrel{i_{*}}{\rightarrow} H_{q}^{\prime}(\tilde{X}) \rightarrow H_{q}(\tilde{X}, N) \stackrel{\partial}{\rightarrow} H_{q-1}^{\prime}(N) \rightarrow \cdots
$$

where $H_{*}^{\prime}(N)$ denotes the homology of the complex $C_{*}^{\prime}(N)$ and similarly for $H_{*}^{\prime}(\tilde{X})$; the symbol $H_{q}(\tilde{X}, N)$ denotes $H_{q}(\tilde{X}, N ; \mathbb{Z})$.

Applying Corollary 14 to the subcomplex $C_{*}^{\prime}(N) \subset C_{*}^{\prime}(\tilde{X})$ we find a group element $g \in H_{0}$ such that $\operatorname{Ker}\left[i_{*}: H_{q-1}^{\prime}(N) \rightarrow H_{q-1}^{\prime}(\tilde{X})\right]$ coincides with $\operatorname{Ker}\left[g_{*}: H_{q-1}^{\prime}(N) \rightarrow\right.$ $\left.H_{q-1}^{\prime}(N)\right]=\operatorname{Ker}\left[j_{*}: H_{q-1}^{\prime}(N) \rightarrow H_{q-1}^{\prime}\left(g^{-1} N\right)\right]$. Here $j: N \rightarrow g^{-1} N$ is the inclusion. Setting $N^{\prime}=g N \subset N$ we obtain

$$
\operatorname{Ker}\left[i_{*}: H_{q-1}^{\prime}\left(N^{\prime}\right) \rightarrow H_{q-1}^{\prime}(\tilde{X})\right]=\operatorname{Ker}\left[i_{*}: H_{q-1}^{\prime}\left(N^{\prime}\right) \rightarrow H_{q-1}^{\prime}(N)\right] .
$$

Now, consider the following commutative diagram:

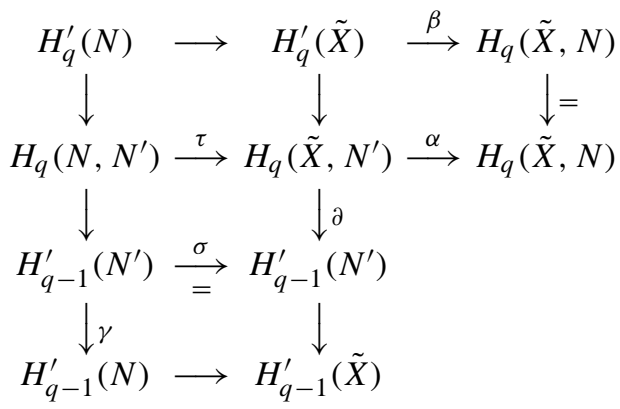

Clearly $\operatorname{Im} \beta \subset \operatorname{Im} \alpha$. The inverse inclusion $\operatorname{Im} \alpha \subset \operatorname{Im} \beta$ would follow once we know that for any $x \in H_{q}\left(\tilde{X}, N^{\prime}\right)$ there exists $y \in H_{q}\left(N, N^{\prime}\right)$ such that $\partial \tau(y)=\partial(x) \in H_{q-1}^{\prime}\left(N^{\prime}\right)$. Now, equality 25 says that $\gamma \circ \sigma^{-1} \circ \partial=0$ is trivial, which (using exactness properties of the diagram above) means that for any $x \in H_{q}\left(\tilde{X}, N^{\prime}\right)$ an element $y \in H_{q}\left(N, N^{\prime}\right)$ with the above mentioned property exists. This shows that $\operatorname{Im} \alpha=\operatorname{Im} \beta$, i.e.

$$
\operatorname{Im}\left[H_{q}\left(\tilde{X}, N^{\prime}\right) \rightarrow H_{q}(\tilde{X}, N)\right]=\operatorname{Im}\left[H_{q}^{\prime}(\tilde{X}) \rightarrow H_{q}(\tilde{X}, N)\right] .
$$

For any neighbourhood of infinity $N^{\prime \prime} \subset N^{\prime}$ one has the following diagram

$$
\begin{aligned}
& H_{q}\left(\tilde{X}, N^{\prime \prime}\right) \longrightarrow H_{q}\left(\tilde{X}, N^{\prime}\right) \\
& \uparrow \quad \gamma \searrow \downarrow \alpha \\
& H_{q}^{\prime}(\tilde{X}) \underset{\beta}{\longrightarrow} H_{q}(\tilde{X}, N)
\end{aligned}
$$

which gives $\operatorname{Im} \beta \subset \operatorname{Im} \gamma \subset \operatorname{Im} \alpha$; but since we already know that $\operatorname{Im} \alpha$ and $\operatorname{Im} \beta$ coincide we obtain $\operatorname{Im} \gamma=\operatorname{Im} \alpha$, i.e. 23. 
This completes the proof of Proposition 15 for the specially chosen neighbourhood $N$. If $N_{1} \subset \tilde{X}$ is an arbitrary neighbourhood of infinity with respect to $\xi$ then $g_{1} N \subset N_{1}$ and we easily see that for any $N^{\prime \prime} \subset g_{1} N^{\prime}$ one has $\operatorname{Im}\left[H_{q}\left(\tilde{X}, N^{\prime \prime}\right) \rightarrow H_{q}\left(\tilde{X}, N_{1}\right)\right]=$ $\operatorname{Im}\left[H_{q}\left(\tilde{X}, g_{1} N^{\prime}\right) \rightarrow H_{q}\left(\tilde{X}, N_{1}\right)\right]$, i.e. 23 is satisfied.

\section{Part III: Cohomological estimates for $\operatorname{cat}^{1}(X, \xi)$}

In Part III we combine the results of Parts I and II to obtain new cohomological lower bounds for $\operatorname{cat}^{1}(X, \xi)$. This allows us to compute explicitly cat ${ }^{1}(X, \xi)$ in some examples. Finally, we compare $\operatorname{cat}^{1}(X, \xi)$ with the values of a $\operatorname{similar}$ invariant $\operatorname{cat}(X, \xi)$ and conclude that their difference can be arbitrarily large.

\section{Line bundles, algebraic integers and movability of homology classes}

Let $X$ be a finite cell complex and $\xi \in H^{1}(X ; \mathbb{R})$ be a nonzero cohomology class. $\xi$ determines the obvious homomorphism $H_{1}(X ; \mathbb{Z}) \rightarrow \mathbb{R}$. Its kernel will be denoted $\operatorname{Ker}(\xi)$. The factor group $H=H_{1}(X ; \mathbb{Z}) / \operatorname{Ker}(\xi)$ is a finitely generated free abelian group which is naturally isomorphic to the group of periods of $\xi$. The rank of $H$ is equals the rank of the class $\xi$; it is denoted by $r=\operatorname{rk}(\xi)$. Consider the covering $p: \tilde{X} \rightarrow X$ corresponding to $\operatorname{Ker}(\xi)$. This covering has $H$ as the group of covering transformations.

Let $\mathcal{V}_{\xi}=\left(\mathbb{C}^{*}\right)^{r}=\operatorname{Hom}\left(H, \mathbb{C}^{*}\right)$ denote the variety of all complex flat line bundles $L$ over $X$ such that the induced flat line bundle $p^{*} L$ over $\tilde{X}$ is trivial. If $t_{1}, \ldots, t_{r} \in H$ is a basis, then the monodromy of $L \in \mathcal{V}_{\xi}$ along $t_{i}$ is a nonzero complex number $x_{i} \in \mathbb{C}^{*}$ and the numbers $x_{1}, \ldots, x_{r} \in \mathbb{C}^{*}$ form a coordinate system on $\mathcal{V}_{\xi}$. Given a flat line bundle $L \in \mathcal{V}_{\xi}$ the monodromy representation of $L$ is the ring homomorphism

$$
\operatorname{Mon}_{L}: \mathbb{Z}[H] \rightarrow \mathbb{C}
$$

sending each $t_{i} \in H$ to $x_{i} \in \mathbb{C}^{*}$.

The dual bundle $L^{*} \in \mathcal{V}_{\xi}$ is such that $L \otimes L^{*}$ is trivial; if $x_{1}, \ldots, x_{r} \in \mathbb{C}^{*}$ are coordinates of $L$ then $x_{1}^{-1}, \ldots, x_{r}^{-1} \in \mathbb{C}^{*}$ are coordinates of $L^{*}$.

Any nonzero element $P \in \mathbb{Z}[H]$ can be written as $P=\sum_{i=1}^{k} a_{i} h_{i}$ where $a_{i} \in \mathbb{Z}$, $a_{i} \neq 0, h_{i} \in H$ and $\xi\left(h_{1}\right)<\cdots<\xi\left(h_{k}\right)$. The nonzero integer $a_{k}$ is called the $\xi$-top coefficient of $P$.

The following notion was introduced in [6, Definition 1.53].

Definition 9. A flat line bundle $L \in \mathcal{V}_{\xi}$ is called a $\xi$-algebraic integer if the kernel of the monodromy homomorphism $\operatorname{Mon}_{L}: \mathbb{Z}[H] \rightarrow \mathbb{C}$ contains a nonzero polynomial $P \in \mathbb{Z}[H]$ having $\xi$-top coefficient 1 . 
Theorem 6. Let $L \in \mathcal{V}_{\xi}$ be a complex flat line bundle over $X$ which is not a $\xi$-algebraic integer. Suppose that for some $v \in H^{q}(X ; L)$ and $z \in H_{q}(\tilde{X} ; \mathbb{Z})$ one has $\left\langle v, p_{*}(z)\right\rangle \neq$ $0 \in \mathbb{C}$ where $p_{*}: H_{q}(\tilde{X} ; \mathbb{Z}) \rightarrow H_{q}\left(X ; L^{*}\right)$ is the obvious coefficient map. Then the class $z$ is not movable to infinity of $\tilde{X}$ with respect to $\xi$.

Proof. We will show that if a homology class $z \in H_{q}(\tilde{X} ; \mathbb{Z})$ is movable to infinity with respect to $\xi$ then $p_{*}(z)=0 \in H_{q}\left(X ; L^{*}\right)$ for any $L \in \mathcal{V}_{\xi}$ which is not a $\xi$-algebraic integer. This statement clearly implies the theorem.

Let $S_{\xi} \subset \Lambda=\mathbb{Z}[H]$ denote the set of all nonzero Laurent polynomials $P \in \Lambda$ having $\xi$-lowest coefficient 1 . The monodromy homomorphism $\operatorname{Mon}_{L^{*}}: \Lambda \rightarrow \mathbb{C}$ is injective when restricted to $S_{\xi}$ (because of our assumption that $L$ is not a $\xi$-algebraic integer). Hence $\operatorname{Mon}_{L^{*}}: \Lambda \rightarrow \mathbb{C}$ extends to the localized ring $\Lambda_{\xi}=S_{\xi}^{-1} \Lambda$.

The homomorphism $p_{*}: H_{q}(\tilde{X} ; \mathbb{Z}) \rightarrow H_{q}\left(X ; L^{*}\right)$ can be decomposed as

$$
p_{*}: H_{q}(\tilde{X} ; \mathbb{Z})=H_{q}(X ; \Lambda) \stackrel{\alpha}{\rightarrow} H_{q}\left(X ; \Lambda_{\xi}\right) \rightarrow H_{q}\left(X ; L^{*}\right)
$$

and the module in the middle equals $H_{q}\left(X ; \Lambda_{\xi}\right)=S_{\xi}^{-1} H_{q}(\tilde{X} ; \mathbb{Z})$. If $z \in H_{q}(\tilde{X} ; \mathbb{Z})$ is movable to infinity with respect to $\xi$ then $\Delta \cdot z=0$ for some $\Delta \in S_{\xi}$ and hence $\alpha(z)=0$ and $p_{*}(z)=0$.

\section{Definition and properties of $\operatorname{cat}^{1}(X, \xi)$}

Let $X$ be a finite polyhedron and $\xi \in H^{1}(X ; \mathbb{R})$ a cohomology class with real coefficients. Let $\omega$ be a closed 1-form on $X$ representing $\xi$ (see [4] for the formalism of closed 1-forms on topological spaces).

Definition 10. Let $N$ be a positive integer. A subset $A \subset X$ is said to be $N$-movable with respect to $\omega$ if there exists a continuous homotopy $h_{t}: A \rightarrow X, t \in[0,1]$, such that $h_{0}: A \rightarrow X$ is the inclusion and for any point $x \in A$ we have

$$
\int_{h_{1}(x)}^{x} \omega>N
$$

where the integral is calculated along the path $t \mapsto h_{1-t}(x) \in X, t \in[0,1]$.

Recall that for $A \subset X$, $\operatorname{cat}_{X}(A)$ denotes the Lusternik-Schnirelmann category of $A$ in $X$, i.e. the minimal integer $k$ such that $A$ can be covered by $k$ open sets in $X$ each of which is null-homotopic in $X$.

The following notion has been introduced in [7].

Definition 11. Let $X$ be a finite polyhedron and $\xi \in H^{1}(X ; \mathbb{R})$. Fix a closed 1-form $\omega$ in $\xi$. The number $\operatorname{cat}^{1}(X, \xi)$ is the minimal integer $k$ such that there exists a closed subset $A \subset X$ with cat $_{X}(X-A) \leq k$ and such that $A$ is $N$-movable with respect to $\omega$ for any positive integer $N$. 
By reversing the order of quantifiers one obtains another notion originally introduced in [4].

Definition 12. Let $X$ be a finite polyhedron and $\xi \in H^{1}(X ; \mathbb{R})$. Fix a closed 1-form $\omega$ in $\xi$. The number $\operatorname{cat}(X, \xi)$ is the minimal integer $k$ such that for any positive integer $N$ there exists a closed subset $A \subset X$ which is $N$-movable with respect to $\omega$ and such that cat $_{X}(X-A) \leq k$.

It is easy to see that neither $\operatorname{cat}^{1}(X, \xi)$ nor cat $(X, \xi)$ depend on the choice of $\omega$. Furthermore both notions are homotopy invariants of the pair $(X, \xi)$ (see [4, 7]). Another observation is that for $\xi=0$ we get the ordinary Lusternik-Schnirelmann category $\operatorname{cat}(X, \xi)=\operatorname{cat}^{1}(X, \xi)=\operatorname{cat}(X)$.

It follows straightforwardly from the definitions that

$$
\operatorname{cat}(X, \xi) \leq \operatorname{cat}^{1}(X, \xi) \leq \operatorname{cat}(X) .
$$

We show later in this paper that for some pairs $(X, \xi)$ one has

$$
\operatorname{cat}(X, \xi)<\operatorname{cat}^{1}(X, \xi)
$$

and that the difference between $\operatorname{cat}^{1}(X, \xi)$ and cat $(X, \xi)$ can indeed be arbitrarily large.

\section{The main estimate}

Theorem 7. Let $X$ be a finite cell complex and $\xi \in H^{1}(X ; \mathbb{R})$. Let $L \in \mathcal{V}_{\xi}$ be a complex flat line bundle over $X$ which is not a $\xi$-algebraic integer. Assume that for some $u \in$ $H^{q}(X ; L)$ and $z \in H_{q}(X ; \Lambda)$ the evaluation $\left\langle u, p_{*}(z)\right\rangle \in \mathbb{C}$ is nonzero where $p_{*}$ : $H_{q}(X ; \Lambda) \rightarrow H_{q}\left(X ; L^{*}\right)$ is the coefficient homomorphism. Then ${ }^{3}$

$$
\operatorname{cat}^{1}(X, \xi) \geq \operatorname{cwgt}(z)+1 \text {. }
$$

Proof. Set $k=\operatorname{cwgt}(z)$ and assume the contrary, i.e. that $\operatorname{cat}^{1}(X, \xi) \leq k$. Then there exists a closed subset $A \subset X$ with cat ${ }_{X} A \leq k$ such that the complement $F=X-A$ is $N$-movable for any $N>0$ with respect to a closed 1-form $\omega$ on $X$ representing $\xi$. Applying the definition, we find that $z$ can be realized by a singular cycle $c$ in $X-A=F$ with coefficients in the local system $\Lambda$.

Consider the covering $p: \tilde{X} \rightarrow X$ corresponding to $\operatorname{Ker}(\xi)$. Viewed differently, the cycle $c$ is a usual singular cycle in $\tilde{X}$ lying in the set $\tilde{F}=p^{-1}(F)$. Since $F$ is $N$-movable for any $N$ we find that any cycle in $\tilde{F}$ is movable to infinity with respect to $\xi$. Thus we obtain a contradiction with Theorem 6 .

\footnotetext{
3 The group $H_{q}(X ; \Lambda)$ is naturally isomorphic to $H_{q}(\tilde{X} ; \mathbb{Z})$. However, the category weights of $z$ viewed as element of $H_{q}(X ; \Lambda)$ or of $H_{q}(\tilde{X} ; \mathbb{Z})$ are in general different. In inequality 28 the symbol cwgt $(z)$ denotes the category weight of $z$ regarded as an element of $H_{q}(X ; \Lambda)$.
} 
Theorem 8. Let $X$ be a finite cell complex and $\xi \in H^{1}(X ; \mathbb{R})$. Let $L \in \mathcal{V}_{\xi}$ be a complex flat line bundle over $X$ which is not a $\xi$-algebraic integer. Suppose that for an integral homology class $z \in H_{q}(\tilde{X} ; \mathbb{Z})=H_{q}(X ; \Lambda)$ and some cohomology classes $u \in H^{d}(X ; L)$ and $u_{i} \in H^{d_{i}}(X ; \mathbb{C})$, where $d_{i}>0$ for $i=1, \ldots, k$, the evaluation $\left\langle u \cup u_{1} \cup \ldots \cup\right.$ $\left.u_{k}, p_{*}(z)\right\rangle \in \mathbb{C}$ is nonzero. Here $p_{*}(z) \in H_{d}\left(X ; L^{*}\right), q=d+d_{1}+\cdots+d_{k}$. Then

$$
\operatorname{cat}^{1}(X, \xi) \geq \operatorname{cwgt}(z)+k+1 .
$$

Here $\operatorname{cwgt}(z)$ denotes the category weight of $z$ viewed as a homology class of $X$ with local coefficient system $\Lambda$.

Proof. First observe that we may assume that the classes $u_{1}, \ldots, u_{k}$ are integral, i.e. lie in $H^{*}(X ; \mathbb{Z})$. Indeed, the product $\left\langle u \cup u_{1} \cup \cdots \cup u_{k}, p_{*}(z)\right\rangle$ is a multilinear function of $u_{1}, \ldots, u_{k}$; since the integral cohomology classes generate $H^{*}(X ; \mathbb{C})$, vanishing of this function on all integral combinations would imply vanishing in general.

Define $z^{\prime}=p^{*}\left(u_{1} \cup \cdots \cup u_{k}\right) \cap z \in H_{d}(\tilde{X} ; \mathbb{Z})=H_{d}(X ; \Lambda)$. Then

$$
\left\langle u, p_{*}\left(z^{\prime}\right)\right\rangle=\left\langle u \cup u_{1} \cup \cdots \cup u_{k}, p_{*}(z)\right\rangle \neq 0 \in \mathbb{C} .
$$

Applying the previous theorem we find $\operatorname{cat}^{1}(X, \xi) \geq \operatorname{cwgt}\left(z^{\prime}\right)+1$. Now, Theorem 2 gives $\operatorname{cwgt}\left(z^{\prime}\right) \geq k+\operatorname{cwgt}(z)$. This completes the proof.

Remark. Consider the statement of Theorem 8 in the special case $\xi=0$. Then the variety $\mathcal{V}_{\xi}$ contains the trivial line bundle $L=\mathbb{C}$ only and $L=\mathbb{C}$ is not a $\xi$-algebraic integer. Hence Theorem 8 gives the inequality

$$
\operatorname{cat}(X) \geq \operatorname{cwgt}(z)+k+1
$$

under the assumption that

$$
\left\langle u_{1} \cup \cdots \cup u_{k}, z\right\rangle \neq 0
$$

where $u_{i} \in H^{d_{i}}(X ; \mathbb{C}), d_{i}>0$ and $z \in H_{d}(X ; \mathbb{C}), d=d_{1}+\cdots+d_{k}$. This claim is a special case of (8).

Example 4. Let $X=\Sigma$ be a closed orientable surface of genus $g>1$ and $\xi \neq 0 \in$ $H^{1}(X ; \mathbb{R})$. Fix a flat line bundle $L \in \mathcal{V}_{\xi}$ which is transcendental (see [9, §6]). Then $H^{1}(X ; L)$ has dimension $2 g-2>0$. Pick a nonzero class $u \in H^{1}(X ; L)$. By Proposition 6.5 from [9] there exists a homology class $z \in H_{1}(X ; \Lambda)$ such that $\left\langle u, p_{*}(z)\right\rangle \neq 0$. Since $\operatorname{cwgt}(z) \geq \operatorname{swgt}(z) \geq 1$ we get $\operatorname{cat}^{1}(\Sigma, \xi) \geq 2$ by applying Theorem 7 . Since cat $^{1}(X, \xi) \leq \operatorname{dim} X$ in general for $\xi \neq 0$, we find

$$
\operatorname{cat}^{1}(\Sigma, \xi)=2
$$

for any nonzero $\xi \in H^{1}(\Sigma ; \mathbb{R})$.

Note that $\operatorname{cat}(\Sigma, \xi)=1$ for any $\xi \neq 0$ (see Theorem 12 in [9]). This gives a first instance where

$$
\operatorname{cat}(X, \xi)<\operatorname{cat}^{1}(X, \xi)
$$




\section{A controlled version of $\operatorname{cat}^{1}(X, \xi)$}

We have seen in Example 4 that $\operatorname{cat}(X, \xi)$ and $\operatorname{cat}^{1}(X, \xi)$ can indeed be different. In order to show that the difference between them can be arbitrarily large, we have to introduce a controlled version of $\operatorname{cat}^{1}(X, \xi)$ which behaves better under cartesian products. The following discussion is very similar to [9, Section 9].

Let $\omega$ be a continuous closed 1-form on a finite cell complex $X$. Let $\xi=[\omega] \epsilon$ $H^{1}(X ; \mathbb{R})$ be the cohomology class represented by $\omega$.

Definition 13. Let $N$ and $C$ be two positive integers. A subset $A \subset X$ is $N$-movable with respect to $\omega$ with control $C$ if there exists a continuous homotopy $h_{t}: A \rightarrow X, t \in[0,1]$, such that (1) $h_{0}: A \rightarrow X$ is the inclusion; (2) for any point $x \in A$ one has

$$
\int_{x}^{h_{1}(x)} \omega<-N
$$

where the integral is calculated along the path $t \mapsto h_{t}(x) \in X, t \in[0,1]$, and (3) for any point $x \in A$ and for any $t \in[0,1]$ one has

$$
\int_{x}^{h_{t}(x)} \omega \leq C
$$

Definition 14. Fix a closed 1-form $\omega$ representing $\xi$. The number $\operatorname{ccat}^{1}(X, \xi)$ is the minimal integer $k$ with the property that there exists $C>0$ and a closed subset $A \subset X$ with cat $_{X}(X-A) \leq k$ such that $A$ is $N$-movable with control $C$ with respect to $\omega$ for every positive integer $N$.

Lemma 16. The following properties hold for $\operatorname{ccat}^{1}(X, \xi)$.

(1) We have $\operatorname{cat}^{1}(X, \xi) \leq \operatorname{ccat}^{1}(X, \xi)$.

(2) If $X$ is connected and $\xi \neq 0$, then ccat $^{1}(X, \xi) \leq \operatorname{cat}(X)-1$.

(3) If $\xi=0$, then $\operatorname{ccat}^{1}(X, \xi)=\operatorname{cat}(X)$.

(4) If $\phi: Y \rightarrow X$ is a homotopy equivalence and $\xi \in H^{1}(X ; \mathbb{R})$, then

$$
\operatorname{ccat}^{1}(X, \xi)=\operatorname{ccat}^{1}\left(Y, \phi^{*} \xi\right) .
$$

Proof. The first assertion is obvious, the remaining assertions are obtained by repeating the arguments given in [4] and [9].

Remark 1. It is worth pointing out that the applications of $\operatorname{cat}^{1}(X, \xi)$ to dynamics described in [7] also hold with the potentially larger quantity $\operatorname{ccat}^{1}(X, \xi)$ (cf. [9, Remark 9.9]).

The desired product inequality now reads as follows. 
Theorem 9. Let $X$ and $Y$ be finite cell complexes and let $\xi_{X} \in H^{1}(X ; \mathbb{R})$ and $\xi_{Y} \in$ $H^{1}(Y ; \mathbb{R})$ be real cohomology classes. Assume that

$$
\operatorname{ccat}^{1}\left(X, \xi_{X}\right)>0 \text { or } \operatorname{ccat}^{1}\left(Y, \xi_{Y}\right)>0 .
$$

Then

$$
\operatorname{ccat}^{1}(X \times Y, \xi) \leq \operatorname{ccat}^{1}\left(X, \xi_{X}\right)+\operatorname{ccat}^{1}\left(Y, \xi_{Y}\right)-1,
$$

where

$$
\xi=\xi_{X} \times 1+1 \times \xi_{Y}
$$

We skip the proof since it is fully analogous to the proof of the similar statement for $\operatorname{ccat}(X, \xi)$ given in [9, Theorem 9].

\section{Calculation of cat $^{1}(X, \xi)$ for products of surfaces}

Theorem 10. Let $M^{2 k}$ denote the product $\Sigma_{1} \times \cdots \times \Sigma_{k}$ where each $\Sigma_{i}$ is a closed orientable surface of genus $g_{i}>1$. Given a cohomology class $\xi \in H^{1}\left(M^{2 k} ; \mathbb{R}\right)$, one has

$$
\operatorname{cat}^{1}\left(M^{2 k}, \xi\right)=\operatorname{ccat}^{1}\left(M^{2 k}, \xi\right)=1+k+r
$$

where $r$ denotes the number of indices $i \in\{1, \ldots, k\}$ such that the cohomology class $\left.\xi\right|_{\Sigma_{i}} \in H^{1}\left(\Sigma_{i} ; \mathbb{R}\right)$ vanishes. In particular

$$
\operatorname{cat}^{1}\left(M^{2 k}, \xi\right)=\operatorname{ccat}^{1}\left(M^{2 k}, \xi\right)=1+k
$$

assuming that $\left.\xi\right|_{\Sigma_{i}} \neq 0 \in H^{1}\left(\Sigma_{i} ; \mathbb{R}\right)$ for any $i=1, \ldots, k$.

Proof. After rearranging the surfaces we may assume that $\xi_{i}=\left.\xi\right|_{\Sigma_{i}}$ is nonzero for $i=$ $1, \ldots, k-r$ and $\xi_{i}=0$ for $i>k-r$.

Note that $\operatorname{ccat}^{1}\left(\Sigma_{i}, \xi_{i}\right)>0$ for any $i=1, \ldots, k$. Indeed, otherwise applying Theorem 10 of [9] we would get $\chi\left(\Sigma_{i}\right)=0$, contradicting our assumption $g_{i}>0$. Hence we may apply the inequality of Theorem 9 several times to obtain

$$
\operatorname{ccat}^{1}\left(M^{2 k}, \xi\right) \leq \sum_{i=1}^{k} \operatorname{ccat}^{1}\left(\Sigma_{i}, \xi_{i}\right)-(k-1) .
$$

By Example 4 and Lemma 16 we have

$$
\operatorname{ccat}^{1}\left(\Sigma_{i}, \xi_{i}\right)=\operatorname{cat}^{1}\left(\Sigma_{i}, \xi_{i}\right)= \begin{cases}2 & \text { if } i \leq k-r, \\ 3 & \text { if } i>k-r,\end{cases}
$$

and thus

$$
\operatorname{ccat}^{1}\left(M^{2 k}, \xi\right) \leq 2(k-r)+3 r-(k-1)=k+r+1 .
$$


Next we prove the opposite inequality to (39). Let $L \in \mathcal{V}_{\xi}$ be transcendental. Define $H=\pi_{1}(M) / \operatorname{Ker}(\xi)$ and $L_{i}=\left.L\right|_{\Sigma_{i}}$ and $H_{i}=\pi_{1}\left(\Sigma_{i}\right) / \operatorname{Ker}\left(\xi_{i}\right)$. It follows that $L_{i}$ is also transcendental. Choose $u_{i}^{\prime} \in H^{1}\left(\Sigma_{i} ; L_{i}\right)$ and $z_{i} \in H_{1}\left(\tilde{\Sigma}_{i} ; \mathbb{Z}\right)$ such that $\left\langle u_{i}^{\prime}, p_{*}\left(z_{i}\right)\right\rangle \neq 0$ as in Example 4 Here $p_{i}: \tilde{\Sigma}_{i} \rightarrow \Sigma_{i}$ is the covering space corresponding to $\operatorname{Ker}\left(\xi_{i}\right)$ and $p_{i *}: H_{*}\left(\tilde{\Sigma}_{i} ; \mathbb{Z}\right) \rightarrow H_{i}\left(\Sigma_{i} ; L_{i}^{*}\right)$. Note that for $i>k-r$ we simply have $\tilde{\Sigma}_{i}=\Sigma_{i}$ and $L_{i}=\mathbb{C}$. Now, $\operatorname{Ker}\left(\xi_{1}\right) \times \cdots \times \operatorname{Ker}\left(\xi_{k}\right) \subset \operatorname{Ker}(\xi)$ so there is a covering map

$$
q: \tilde{\Sigma}_{1} \times \cdots \times \tilde{\Sigma}_{k} \rightarrow \tilde{M}
$$

where $\tilde{M}$ is the covering space of $M$ corresponding to $\operatorname{Ker}(\xi)$. Let

$$
\begin{aligned}
z^{\prime} & =z_{1} \times \cdots \times z_{k} \in H_{k}\left(M ; \mathbb{Z}\left[H_{1} \times \cdots \times H_{k}\right]\right) \cong H_{k}\left(\tilde{\Sigma}_{1} \times \cdots \times \tilde{\Sigma}_{k} ; \mathbb{Z}\right), \\
z & =q_{*}\left(z^{\prime}\right) \in H_{k}(M ; \mathbb{Z}[H]) \cong H_{k}(\tilde{M} ; \mathbb{Z}) .
\end{aligned}
$$

It follows from Corollary 8 and Lemma 1 that $\operatorname{cwgt}(z) \geq k$ (where $z$ is viewed as an element of $\left.H_{k}(M ; \mathbb{Z}[H])\right)$.

Define

$$
\begin{aligned}
u & =u_{1}^{\prime} \times \cdots \times u_{k-r}^{\prime} \times 1 \times \cdots \times 1 \in H^{k-r}(M ; L), \\
u_{j} & =p_{k-r+j}^{*} u_{k-r+j}^{\prime} \in H^{1}(M ; \mathbb{C}), \quad j=1, \ldots, r,
\end{aligned}
$$

where $p_{k-r+j}: M \rightarrow \Sigma_{k-r+j}$ is the projection. Notice that

$$
\left\langle u \cup u_{1} \cup \cdots \cup u_{r}, p_{*}(z)\right\rangle= \pm \prod_{i=1}^{k}\left\langle u_{i}^{\prime}, p_{i_{*}}\left(z_{i}\right)\right\rangle \neq 0 .
$$

Theorem 8 and Corollary 8 apply and give

$$
\operatorname{cat}^{1}(M, \xi) \geq \operatorname{cwgt}(z)+r+1 \geq k+r+1 .
$$

Combining this with 39 we obtain

$$
\operatorname{cat}^{1}(M, \xi)=\operatorname{ccat}^{1}(M, \xi)=k+r+1
$$

as claimed.

We now want to compare the values of $\operatorname{cat}^{1}(M, \xi)$ with the invariant cat $(M, \xi)$ (see Definition 12) for products of surfaces $M=\Sigma_{1} \times \cdots \times \Sigma_{k}$ where each $\Sigma_{i}$ is a closed orientable surface of genus $g_{i}>1$. It was shown in [9, Thm. 17] that

$$
\operatorname{cat}(M, \xi)=1+2 r
$$

where $r$ denotes the number of indices $i \in\{1, \ldots, k\}$ such that $\left.\xi\right|_{\Sigma_{i}}=0$.

Corollary 17. Under the assumptions of Theorem 10 the difference

$$
\operatorname{cat}^{1}(M, \xi)-\operatorname{cat}(M, \xi)
$$

equals the number of indices $i \in\{1, \ldots, k\}$ such that $\left.\xi\right|_{\Sigma_{i}} \neq 0 \in H^{1}\left(\Sigma_{i} ; \mathbb{R}\right)$.

Corollary 17 leads to the following statement which is one of the main results of this paper:

Corollary 18. The difference (41) can be arbitrarily large. 


\section{References}

[1] Cornea, O., Lupton, G., Oprea, J., Tanré, D.: Lusternik-Schnirelmann Category. Amer. Math. Soc. (2003) Zbl 1032.55001 MR 1990857

[2] Eisenbud, D.: Commutative Algebra. With a View towards Algebraic Geometry. Springer, New York (1995) Zbl 0819.13001 MR 1322960

[3] Faddell, E., Husseini, S.: Category weight and Steenrod operations. Bol. Soc. Mat. Mexicana 37, 151-161 (1992) Zbl 0848.55001 MR 1317569

[4] Farber, M.: Zeros of closed 1-forms, homoclinic orbits and Lusternik-Schnirelman theory. Topol. Methods Nonlinear Anal. 19, 123-152 (2002) Zbl pre01819656 MR 1921889

[5] Farber, M.: Lusternik-Schnirelman theory and dynamics. In: Lusternik-Schnirelmann Category and Related Topics, Contemp. Math. 316, Amer. Math. Soc., 95-111 (2002) Zbl 1029.58006 MR 1962156

[6] Farber, M.: Topology of Closed One-Forms. Math. Surveys Monogr. 108, Amer. Math. Soc., Providence, RI (2004) Zbl 1052.58016 MR 2034601

[7] Farber, M., Kappeler, T.: Lusternik-Schnirelman theory and dynamics, II. Proc. Steklov Inst. Math. 247, 232-245 (2004) Zbl 1106.58007 MR 2168176

[8] Farber, M., Schütz, D.: Moving homology classes to infinity. Forum Math. 19, 281-296 (2007) MR 2313841

[9] Farber, M., Schütz, D.: Cohomological estimates for cat $(X, \xi)$. Geom. Topol. 11, 1255-1288 (2007) MR 2326945

[10] Krasnosel'skiǔ, M. A.: On special coverings of a finite dimensional sphere. Dokl. Akad. Nauk SSSR 103, 961-964 (1955) (in Russian) MR 0081454

[11] Novikov, S. P.: Multi-valued functions and functionals. An analogue of Morse theory. Soviet Math. Dokl. 24, 222-226 (1981) Zbl 0505.58011 MR 0630459

[12] Novikov, S. P.: The Hamiltonian formalism and a multi-valued analogue of Morse theory. Russian Math. Surveys 37, no. 5, 1-56 (1982) Zbl 0571.58011

[13] Rudyak, Y.: On category weight and its applications. Topology 38, 37-55 (1999) Zbl 0927.55005 MR 1644063

[14] Spanier, E.: Algebraic Topology. McGraw-Hill (1966) Zbl 0145.43303 MR 0210112

[15] Strom, J.: Essential category weight. Preprint (1997)

[16] Weibel, C. A.: An Introduction to Homological Algebra. Cambridge Univ. Press (1994) Zbl 0797.18001 MR 1269324 